\title{
Assessment of Spontaneous Use and Experience of Use of The Affected Upper Limb in 6-Year-Old Infantile Hemiplegia After Applying Combined Intensive Therapy. Case Series
}

\author{
Rocío Palomo Carrión* \\ Physiotherapy Department, CEU San Pablo University, Spain
}

*Corresponding author: Rocío Palomo Carrión PhD, Physiotherapy Department,

CEU San Pablo University, Madrid, Spain.

\section{Abstract}

Introduction: Children with hemiplegia do not use the affected upper limb. This disuse of the upper limb is known as "Disregard development." Therefore, therapy should create the opportunity, experience and environment in which a child can learn to use the affected member. Being the modified Constraint Induced Movement Therapy (mCIMT) and the Bimanual Intensive Therapy carried out at home.

Objective: To assess the increase in spontaneous use of the affected upper limb in congenital hemiplegia childhood 6-10 years with low hand performance after applying a combined intensive therapy protocol.

Method: 3 children ( 6 years old) diagnosed with congenital infantile hemiplegia are recruited to execute a 100-hour combined intensive therapy protocol. 3 measurements are carried out for the variables of spontaneous use and the experience of use of the upper limb.

Results: Increased spontaneous use of 24 AHA units, measured with the Assisting Hand Assessment (AHA) scale after the end of the protocol and for the experience of use of the upper limb, measured by Children's Hand- Use Experience Questionnaire (CHEQ) increases of 11.67 activities executed with both hands.

Conclusion: Children with congenital infantile hemiplegia with low hand performance could obtain a greater increase in spontaneous use executing a protocol of combined intensive therapy.

Key words: Disabled children; Family; Physical therapy specialty; Hemiplegia; House calls; Upper extremity

\section{Introduction}

Infantile Cerebral Palsy (ICP) is defined as a series of permanent disorders that affect the motor and postural development of the Child [1]. Functional limitations in motor activity occur, triggered by brain injury caused during the maturation of the central nervous system of the fetus or infant [2]. ICP is characterized as a nonprogressive encephalopathy. It is a chronic sensorimotor disorder that leads to the alteration of sensitivity, perception, cognition, communication and behavior, as well as causing changes in posture, muscle tone and production of inappropriate movements [3]. The hemiplegia is one of the most common forms of ICP, representing 1 in 1300 live births in developed countries [4]. The first symptoms of early lateralization in infantile hemiplegia are the lack of flexibility in conjunction with the asymmetry and motor spasticity prevalent in the affected upper limb.
If we follow the International Classification of Functioning, Disability and Health (ICF), at the level of body structure and function, children with hemiplegia may present changes in the structure and function of the brain (seen in functional magnetic resonance imaging), resulting in Alterations of spasticity, muscle length, sensation and weakness. Activity limitations are common in areas such as self-care, school and home-related activities [5,6]. Participation can be restricted in the home, school and community life in general and, in turn, can affect the quality of life [7]. Thus, the severity of the deterioration varies widely, depending on the location and brain damage. Decreased upper limb function may be due to sensory abnormalities, weak muscles, lack of selective finger movements, loss of movement speed, lack of motor skills, presence of movements associated and mirror and reduced flexibility due to spasticity [8]. 
From an early age, children do not use the affected upper limb, which causes increased muscle tone, active and passive loss of movement of the upper limb joints and producing a general delay in musculoskeletal maturation [9]. This disuse of the affected upper limb is known as "Disregard of development". Therefore, therapy should create the opportunity, experience and environment in which a child can learn to use his affected member. This experience should reverse the behavioral aspect of the suppression of the use of the affected upper limb and reward the use of that member even in the simplest tasks, such as the stabilization of an object [10].

Thus, in the systematic review of Novak and other researchers [11] on the interventions of greater evidence in ICP, reference is made to those that are based on activity and participation. Focusing on the child's strengths, interests and motivation to allow a better quality of life. Among these therapies of great evidence and reliability are programs designed for home, bimanual intensive therapy (BIT) and Constraint induced movement therapy (CIMT) [12]. CIMT and BIT are two contemporary approaches included in intensive therapies and based on motor learning, which focus directly on addressing the impaired function of the affected upper limb in children with unilateral cerebral palsy [13-15]. CIMT, has its origin in behavioral research with non-human primates, conducted by Taub and his collaborators [16]. It consists of the containment of the healthy upper limb during $90 \%$ of the hours and the execution of activities in progression of difficulty for the affected upper limb, 6 consecutive hours per day in a period of 12 days. In pediatrics modified protocols are used, in which the execution time of the therapy is extended with less daily hours of treatment, being called modified Constraint Induced Movement Therapy (mCIMT) $[17,18]$. The BIT was described in 2006, at the University of Columbia in the EE: UU, a group of researchers led by Dr. Andrew Gordon, consists of the execution of bimanual activities where each hand receives a different role. The home protocol uses a total of 90 hours of BIT in 9 weeks with a total of 2 hours per day from Monday to Friday $[19,20]$.

Both therapies are based on neuroplasticity, cortical reorganization and overcoming the non-use of the affected upper limb by executing structured activities designed to be carried out at home. Thus, they are based on the philosophy of family-centered interventions because they allow parents to remain in the role of "expert" caregiver for their Child [21]. Families can select activities for the inclusion of therapy that are meaningful and motivating for themselves and their children, which results in greater adherence of both parts (parents-children) to treatment [22].

\section{Objective}

To assess the increase in spontaneous use of the affected upper limb in congenital infantile hemiplegia from 6-10 years with low hand performance after applying a protocol of combined intensive therapy.

\section{Materials and Methods}

The ethical approval of the study was obtained by the ethical committee of the Virgen de la Salud Hospital in Toledo. Before entering it, the parents' informed consent of the parents was acquired. Three subjects ( 2 girls and one boy) were obtained from the Virgen de la Salud Hospital following the established inclusion and exclusion criteria.

As inclusion criteria: diagnosis of congenital infantile hemiplegia, age between from 6 to 10 years, lack of use of the affected upper limb and presence or not of grasp, or weak grasp (score 1 or 2 in the flow bimanual ítem into AHA scale), level I-III of the Manual Ability Classification System (MACS) [23] and level I-III within the Gross Motor Function Classification System (GMFCS) [24]. The exclusion criteria would be low cognitive level, presence of contractures, surgery 3 months prior to the intervention, botulinum toxin 3 months prior to or during the intervention, and pharmacologically uncontrolled epilepsy.

The 3 children were diagnosed with congenital infantile hemiplegia with an average age of 6 years, being in a level II within the MACS and a level I within the GMFCS. The 3 subjects completed the intervention protocol successfully, with no abandonment by any family.

\section{Design and procedure}

The results of the different variables were measured in 3 times: at the beginning of the treatment, at 8 weeks and at 10 weeks of combined intensive therapy treatment.

Interventions: A protocol of combined intensive therapy at home was designed with a therapeutic dose of 100 hours for 10 weeks. Considering the study by Sakzewski et al [25] in which an intensive therapy dose of 60 hours or more allows the benefits to be maintained 6 months post-intervention. The protocol consists of 80 hours of mCIMT followed by 20 hours of BIT where structured activities are carried out for 2 hours not continued from Monday to Friday in the 10 weeks. Within the mCIMT, unimanual activities were established and with a progression from proximal to distal. A method of partial containment for the healthy hand is used as a glove, manufactured by each family according to the child's interest (Figure 1). In bimanual activities, each of the hands received a different role, starting first with the affected hand as an assistant hand and then handling.

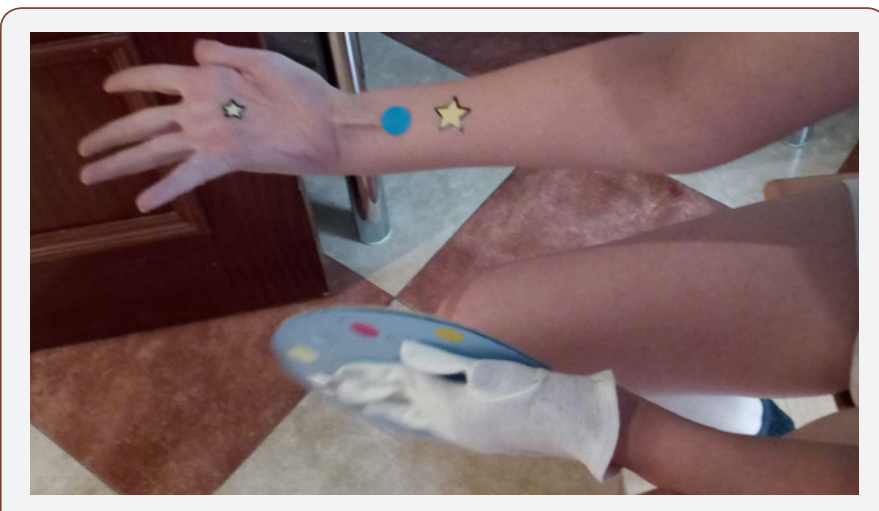

Figure 1: Method of partial containment of the healthy hand.

The parents were instructed by the therapist in the execution of the activities, increasing the parent-child feedback and greater 
adherence to therapy. A weekly follow-up was scheduled, where the therapist had to ensure that the families complied with the execution of activities through a registry, avoid complications and encourage motivation and continuity in the intervention.

Primary measures: The AHA was the tool for measuring the spontaneous use and effectiveness of the affected upper limb in bimanual tasks, validated for children with infantile hemiplegia and obstetrical brachial palsy with an age of 18 months to 12 years [2628]. The play session is recorded on video and scored later. Given the age range of the participants in this study, the School Kids AHA, qualified with version 5.0, will be used, which includes 20 items scored from 0 ("not done") to 4 ("effective use"), valid and reliable.

Secondary measures: The experience of use of the affected upper limb determines if the child involves the affected upper limb when performing structured bimanual tasks and how is the effectiveness of use when the child incorporates it. It allows observing the execution time of the proposed task compared to a child of the same age and the presence of discomfort from the use of his affected hand during the completion of the proposed tasks (29 tasks) [29].

For the qualification of each of the variables to the experience of use of the affected hand, the Children's Hand-use Experience Questionnaire, CHEQ, is used. The CHEQ questionnaire consisting of 29 bimanual activities, aimed at children with unilateral involvement (infantile hemiplegia, Obstetrical brachial palsy, reduced use of one of the upper limbs ...) at an age of 6 to 18 years. It can be answered by the parents, the therapist or the child himself when his cognitive level is adequate, in this study he was answered by the mothers of the 3 children [30].

Data analysis: A descriptive analysis of the data was carried out for the different variables, based on the average value to execute the comparisons between them.

\section{Results and Discussion}

Table 1 shows the average data obtained for the three measurements taken of the variables studied in the different subjects.

Table 1: Spontaneous use measured through the AHA scale in AHA units (0-100), never does the activity, needs help to do the activity, uses one hand, uses both hands (support or grasp) measured through the CHEQ questionnaire in number of activities (0-29 activities), hand use effectiveness, execution time of the bimanual activity and discomfort of the execution of the activity measured through the CHEQ questionnaire with a scale of 1-4.

\begin{tabular}{|c|c|c|c|}
\hline \multicolumn{2}{|c|}{ Assessment 1 } & Assessment 2 & Assessment 3 \\
\hline Affected upper limb spontaneous use & 29,5 & 50 & 55 \\
\hline \multicolumn{2}{|c|}{ CHEQ Questionnaire } \\
\hline Never does the activity & 6,67 & 6,67 & 6,67 \\
\hline Needs help to do the activity & 15 & 6,33 & 5,33 \\
\hline Uses one hand (healthy hand) & 2 & 16 & 17 \\
\hline Uses both hands & 5,33 & 16 & 17 \\
\hline Uses grasp & 3,67 & 0 & 0 \\
\hline Uses support & 1,67 & 2,30 & 2,32 \\
\hline Hand use effectiveness & 1,47 & 2,07 & 2,20 \\
\hline Activity execution time & 1,33 & 2 & 2 \\
\hline discomfort of execution of the activity & 1,30 & & 17 \\
\hline
\end{tabular}

\section{Primary results}

The greatest increase obtained for the spontaneous use of the affected upper limb was appreciated from the first to the third measurement with a total increase of 25,5 AHA units (produced by the increase of 20.5 AHA units of the 1-2 assessment). From the second to the third, during the execution of the 20 hours of BIT there was an increase of 5 AHA units. Among all the assessments, clinically relevant gains were produced since there was an increase $=/>$ to 5 AHA units.

\section{Secondary results}

A stability is appreciated for the values obtained in the activities not carried out during the three assessments. The activities carried out with help are reduced from the first assessment, reaching a total of 5.33 activities in the last assessment. The number of activities carried out with one hand is reduced between valuations 1-2 and reaching 0 activities in valuation 4 . There is an increase in activities carried out with both hands (healthy and affected), from 5.33 activities in the first assessment to a total of 17 activities for the third assessment. No support is used from valuation 2 for the execution of the activities carried out with both hands and is corresponding to the increase in the use of the clamp from valuation 1 to valuation 3 (executing 17 activities with grasp on the affected hand). The effectiveness of hand use has an increase for the three valuations, its value being 0.85 points from valuation 1 to valuation 3.

The time score along the 3 valuations acquires an increase of 0.87 points from valuation 1 to valuation 3 . The value obtained for each of the four valuations for the discomfort of executing the activity increases since the valuation 1-2, keeping the value stable with a score of 2 for valuation 3.

The functional gains obtained in the affected upper limb for the children in our study were manifested in an increase in spontaneous 
use, faster start of use of the affected hand and amplitude of forearm movement, as well as the possibility of reaching and improving the quality of the object's support by the affected hand. These functional improvements are also found in the study by Reidy et al, in which a combined intensive therapy protocol of 114 hours of mCIMT was carried out followed by 12 hours of BIT in children with infantile hemiplegia of low bimanual functional performance. In our study, the increase AHA units Results was higher than the children in the study by Reydi et al. [31], although a lower dose of mCIMT was used. This suggests that the etiology of the lesion and the baseline situation of hand performance could influence the results. Since in the study that has been designed all children were diagnosed with congenital hemiplegia and in addition, the initial situation of hand performance was lower than that of the study by Reydi et al [31]. While in the latter, the children had a diagnosis of hemiparesis with a heterogeneous etiology. The influence of the etiology of the brain injury on the results of the functionality of the upper limb is demonstrated in the research of Feys et al [32], in which it was studied that children with pure periventricular lesions achieved clinically relevant improvements compared to mixed lesions. The differences between congenital cortical-subcortical lesions and acquired lesions were not significant. In addition, infarction of the middle cerebral artery and lesions in the basal ganglia (thalamic lesions) were significantly correlated with worse performance of the affected upper limb. The type of injury determined by brain damage and the location of the lesion could influence the results of children with hemiplegia respect of the function in the upper limb. This is also confirmed in the study by Nordstrand et al [33] in 2015 when applying a mCIMT protocol in infants aged 3 to 8 months, in which the children with periventricular lesion obtained better results and developed less probabilities of acquiring a low hand performance (when they reached the age of 2 years) unlike the children with infarction of the middle cerebral artery) [33].

The functional gains obtained in the spontaneous use of the affected hand observed in the AHA scale, are represented in the CHEQ questionnaire where greater effectiveness is observed in hand use, as well as in the reduction of the execution time of the task, also due to the independence of bimanual activities due to upper limb affected participation by a greater number of tasks performed with two hands (increase of 10.67 activities). Therefore, it could be said that the execution of an unimanual intervention such as mCIMT in children with low hand performance would induce bimanual changes, without the need to carry out explicitly bimanual work to favor the inclusion of the affected upper limb. Bimanual capacity transfers after unimanual work were studied in the research of Chaleat-Valayer et al [34] Where a very high Pearson linear correlation coefficient between unimanual and bimanual capacity of $r=0.87$ was acquired, with a statistically significant relationship ( $\mathrm{p}<0.01$ ) between unimanual work and bimanual functional gains in the upper limb affected.

In addition, it could be thought that children diagnosed with congenital hemiplegia with low hand performance acquire a greater satisfaction of performing the bimanual task, reducing their discomfort possibly correlated with the increase in their spontaneous use and overcoming the frustration [35].

Whose functioning continues to increase and with functional gains until the end of the 10 weeks of structured intervention. The increase in spontaneous use favors the independence and greater participation of the affected upper limb in daily activities, as the CHEQ questionnaire shows. In addition, a possible relationship between uni and bimanual capacity can be seen after applying mCIMT and the frustration of involvement of the affected upper limb is reduced as spontaneous use increases, when hand performance is low.

The limitations of the study are based on the lack of a large sample of subjects and control group to be able to affirm the effectiveness of the therapy and to be able to generalize the data obtained. Therefore, it would be important to continue future research on these guidelines through randomized clinical trials with a control group.

\section{Conclusion}

Children with congenital hemiplegia (6 years old) with low hand performance may obtain a greater increase in spontaneous use, executing a protocol of combined intensive therapy with 100 hours of dosage.

\section{Acknowledgement}

Thank the families and children who did the combined intensive protocol because their work was excellent and met the times an all the activities proposed successfully.

\section{Conflict of Interest}

There is no conflict of interest by the author.

\section{References}

1. Bax M, Goldstein M, Rosenbaum P, Leviton A, Paneth N, et al. (2005) Proposed definition and classification of cerebral palsy, April 2005. Dev Med Child Neurol 47(8): 571-576.

2. Stanley F, Blair E, Alberman E (2000) Cerebral Palsies: Epidemiology and Causal Pathways. London, England: Mac Keith Press.

3. Bringas-Grande A, Fernández-Luque A, García-Alfaro C, Barrera-Chacón M, ToledoGonzález M, et al. (2002) [Cerebral palsy in childhood: 250 cases report]. Rev Neurol 35(9): 812-817.

4. Robaina-Castellanos GR, Riesgo-Rodriguez S, Robaina-Castellanos MS (2007) [Definition and classification of cerebral palsy: a problem that has already been solved?]. Rev Neurol 45(2): 110-117.

5. Chen CY, Tafone S, Lo W, Heathcock JC (2015) Perinatal stroke causes abnormal trajectory and laterality in reaching during early infancy. Res Dev Disabil 38: 301-308.

6. Camacho-Salas A, Pallás-Alonso CR, de la Cruz-Bértolo J, Simón-de Las Heras R, Mateos-Beato F (2007) Cerebral palsy: the concept and population-based registers. Rev Neurol 45(8): 503-508.

7. Hoare B, Imms C, Randall M, Carey L (2011) Linking cerebral palsy upper limb measures to the International Classification of Functioning, Disability and Health. J Rehabil Med 43(11): 987-996.

8. Burridge JH, Turk R, Notley SV, Pickering RM, Simpson DM (2009) The relationship between upper limb activity and impairment in post-stroke hemiplegia. Disabil Rehabil 31(2): 109-117.

9. Klevberg GL, Østensjø S, Krumlinde-Sundholm L, Elkjær S, Jahnsen RB (2017) Hand Function in a Population-Based Sample of Young Children 
with Unilateral or Bilateral Cerebral Palsy. Phys Occup Ther Pediatr 37(5): 528-540.

10. Kuhtz-Buschbeck JP, Krumlinde-Sundholm L, Eliasson A-C, Forssberg H (2000) Quantitative assessment of mirror movements in children and adolescents with hemiplegic cerebral palsy. Developmental Medicine and Child Neurology 42(11): 728-736.

11. Deluca SC, Echols K, Law CR, Ramey SL (2006) Intensive pediatric constraint-induced therapy for children with cerebral palsy: randomized, controlled, crossover trial. J Child Neurol 21(11): 931-938.

12. Novak I, McIntyre S, Morgan C, Campbell L, Dark L, et al (2013) A systematic review of interventions for children with cerebral palsy: state of the evidence. Dev Med Child Neurol 55(10): 885-910.

13. Gordon AM, Hung YC, Brandao M, Ferre CL, Kuo HC, et al. (2011) Bimanual training and constraint-induced movement therapy in children with hemiplegic cerebral palsy: a randomized trial. Neurorehabil Neural Repair 25(8): 692-702.

14. Sakzewski L, Ziviani J, Abbott DF, Macdonell RA, Jackson GD, et al. (2011) Randomized trial of constraint-induced movement therapy and bimanual training on activity outcomes for children with congenital hemiplegia. Dev Med Child Neurol 53(4): 313-320.

15. Aarts PB, Jongerius PH, Geerdink YA, van Limbeek J, Geurts AC (2010) Effectiveness of modified constraint-induced movement therapy in children with unilateral spastic cerebral palsy: a randomized controlled trial. Neurorehabil Neural Repair 24(6): 509-518.

16. Taub E, Harger M, Grier HC, Hodos W (1980) Some anatomical observations following chronic dorsal rhizotomy in monkeys. Neuroscience 5(2): 389-401.

17. Charles J, Gordon AM (2005) A critical review of constraint-induced movement Therapy and forced use in children with hemiplegia. Neural Plast 12(2-3): 245-61.

18. Eliasson AC, Krumlinde-sundholm L, Shaw K, Wang C (2005) Effects of constraint-induced movement therapy in young children with hemiplegic cerebral palsy: an adapted model. Dev Med Child Neurol 47(4): 266-275.

19. Charles J, Gordon AM (2006) Development of hand-arm bimanual intensive training (HABIT) for improving bimanual coordination in children with hemiplegic cerebral palsy. Dev Med Child Neurol 48(11): 931-936.

20. Gordon AM, Schneider JA, Chinnan A, Charles JR (2007) Efficacy of a hand-arm bimanual intensive therapy (HABIT) in children with hemiplegic cerebral palsy a randomized control trial. Dev Med Child Neurol 49(11): 830-838.

21. Eliasson AC, Shaw K, Berg E, Krumlinde-Sundholm L (2011) An ecological approach of Constraint Induced Movement Therapy for 2-3-year-old children: a randomized control trial. Res Dev Disabil 32(6): 2820-2828.

22. Facchin P, Rosa-Rizzotto M, Visonà Dalla Pozza L, et al. (2011) Multisite trial comparing the efficacy of constraint-induced movement therapy with that of bimanual intensive training in children with hemiplegic cerebral palsy: postintervention results. Am J Phys Med Rehabil 90(7): 539-553.

23. Eliasson AC, Krumlinde-Sundholm L, Rösblad B, Beckung E, Arner M, et al. (2006) The Manual Ability Classification System (MACS) for children withcerebral palsy: scale development and evidence of validity and reliability. Dev Med Child Neurol 48(7): 549-554.

24. Palisano R, Rosenbaum P, Walter S, Russell D, Wood E, et al. (1997) Development and reliability of a system to classify gross motor function in children with cerebral palsy. Dev Med Child Neurol 39(4): 214-223.

25. Sakzewski L, Provan K, Ziviani J, Boyd RN (2015) Comparison of dosage of intensive upper limb therapy for children with unilateral cerebral palsy: how big should the therapy pill be? Res Dev Disabil 37: 9-16.

26. Holmefur MM, Krumlinde-Sundholm L (2016) Psychometric properties of a revised version of the Assisting Hand Assessment (Kids-AHA 5.0). Dev Med Child Neurol 58(6): 618-624.

27. Krumlinde-Sundholm L, Holmefur M, Kottorp A, Eliasson AC (2007) The assisting hand assessment: current evidence of validity, reliability, and responsiveness to change. Dev Med Child Neurol 49: 259-264.

28. Holmefur M, Aarts P, Hoare B, Krumlinde-Sundholm L (2009) Testretest and alternate forms reliability of the assisting hand assessment. J Rehabil Med 41: 886-891.

29. Sköld A, Hermansson LN, Krumlinde-Sundholm L, Eliasson AC (2011) Development and evidence of validity for the Children's Hand-use Experience Questionnaire (CHEQ). Dev Med Child Neurol 53(5): 436442.

30. Amer A, Eliasson AC, Peny-Dahlstrand M, Hermansson L (2016) Validity and test-retest reliability of Children's Hand-use Experience Questionnaire in children with unilateral cerebral palsy. Dev Med Child Neurol 58(7): 743-749.

31. Reidy TG, Naber E, Viguers E, Allison K, Brady K, et al. (2012) Outcomes of a clinic-based pediatric constraint-induced movement therapy program. Phys Occup Ther Pediatr. 32(4): 355-367.

32. Feys H, Eyssen M, Jaspers E, Klingels K, Desloovere K, Molenaers G, et al. (2010) Relation between neuroradiological findings and upper limb function in hemiplegic cerebral palsy. Eur J Paediatr Neurol 14(2):169777.

33. Nordstrand L, Holmefur M, Kits A, Eliasson AC (2015) Improvements in bimanual hand function after baby-CIMT in two-year old children with unilateral cerebral palsy: A retrospective study. Res Dev Disabil 41-42: 86-93.

34. Chaleat-Valayer E, Bard-Pondarre R, Ganne C, Roumenoff F, Combey A, et al. (2015) Relation between unimanual capacities and bimanual performance in hemiplegic cerebral-palsied children: impact of synkinesis. Eur J Paediatr Neurol 19(2): 193-201.

35. Cohen-Holzer M, Sorek G, Kerem J, Katz-Leurer M (2017) The impact of combined constraint-induced and bimanual arm training program on the perceived hand-use experience of children with unilateral cerebral palsy. Dev Neurorehabil 20(6): 355-360. 